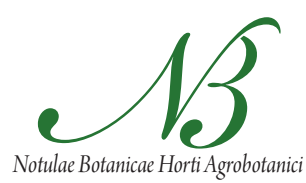

Cluj-Napoca

\title{
Seasonal Pattern of Flavonoid Content and Related Enzyme Activities in Leaves of Ginkgo biloba L.
}

\author{
Shuiyuan CHENG ${ }^{1}$, Feng XU², Linling $\mathrm{LI}^{1}$, Hua CHENG ${ }^{1}$, Weiwei ZHANG ${ }^{3}$ \\ ${ }^{1}$ Hubei Key Laboratory of Economic Forest Germplasm Improvement and Resources Comprehensive Utilization, \\ Huanggang Normal University, Huanggang 438000, China; s_y_cheng@sina.com \\ ${ }^{2}$ College of Horticulture and Gardening, Yangtze University, Jingzhou 434025, China \\ ${ }^{3}$ College of Forest Resources and Environment, Nanjing Forestry University, Nanjing 210037, China
}

\begin{abstract}
The contents of flavonoids and other related components, in addition to the activities of related enzymes, such as phenylalanine ammonia-lyase (PAL), peroxidase (POD), chalcone synthase (CHS), and UDP-glucose:flavonoid 3-O-glucosyltransferase (UFGT), were determined in Ginkgo biloba L. leaves during leaf development. The results indicated that POD activity increased gradually in the early stages and reached its first activity peak in the beginning of Jul., the second one in late Aug., and the third one (the highest peak) in the middle of Oct. The changes in POD activity were irregular, and its activity was maintained at a low level in the early stages. However, it increased rapidly from the middle of Sep., and reached its highest value in the middle of Oct. Three peaks were obtained for PAL activity. The first peak, with the highest value was in early to middle Jul. The second one, with the lowest value, was in late Aug., and the last one, with a higher value and the longest period, was after the middle of Sep. UFGT activity had a speedy increase in the early stages and continued to increase after May. However, the net increase remained low. CHS activity was enhanced during all stages and showed two obvious peaks. The first time was in early Jul., and the other, with a higher value, was in the middle of Nov. Although PAL activity had a close relationship with the flavonoid content, no notable correlation could be identified between them. A significant correlation between CHS activity and the flavonoid content showed that CHS was one of the key enzymes involved in flavonoid biosynthesis. The relationship between flavonoid content and other related enzymes is discussed in this study.
\end{abstract}

Keywords: enzymes, flavonoids, Ginkgo biloba, leaf

Abbreviation: $\mathrm{C} 4 \mathrm{H}=$ Cinnamated-4-hydroxylase; $\mathrm{CAD}=$ cinnamatod-alcohol dehydrogenase; $\mathrm{CHI}=\mathrm{Chalcone}$ isomerase; $\mathrm{CHS}=$ Chalcone synthase; $4 \mathrm{CL}=4$-coumanate $\mathrm{COA}$ ligase; $\mathrm{FB}=$ flavonoids biosynthesis; $\mathrm{FC}=$ flavonoids content; $\mathrm{FF}=$ flavonoids formation; $\mathrm{F} 3 \mathrm{H}=$ flavanone-3-hydroxylase; $\mathrm{PAL}=$ phenylalanineammonia-lyase $\mathrm{PAS}=$ phenylalanine synthase $\mathrm{POD}=\mathrm{Peroxidase} ; \mathrm{PPO}=\mathrm{Polyphenolic}$ oxidase; UFGT=glucose:flavonoid; UDP=3-O-glucosyltransferase

\section{Introduction}

Currently, Ginkgo biloba L. is one of the most popular functional plants, especially used as a medicinal plant. Extracts of G. biloba leaves contain some active compounds, such as flavonoids and terpene lactones, and can therefore increase peripheral and cerebral blood flows (Rice-Evans et al., 1997; Smith and Luo, 2004). Numerous studies have focused on $G$. biloba leaf because flavonoid content (FC) in G. biloba leaf is higher than that in G. biloba seed. Thus far, approximately 38 types of flavonoids present in the leaf have been isolated (van Beek and Montoro, 2009). In recent years, some reports have been published the effects of environmental and endogenous factors on FC and biosynthetic mechanism of flavonoid formation (FF), as well as tissue culture techniques for producing flavonoids (Cheng et al., 2009; Hao et al., 2010). According to the knowledge gathered from other plant systems, more than 10 enzymes take part in the conversion process from phe- nylalanine to flavonoids in a plant. However, no direct data on flavonoid biosynthesis (FB) in G. biloba leaves are available, and only the changes of phenylalanine ammonia-lyase (PAL) activity in G. biloba leaf has been studied so far (Xu et al., 2008a).

Although studies on FF have been carried out in the past and great progress has been made in this field, reports on FF based on G. biloba have not been published yet. However, the basic process of FF is clear from studies on other plants. The modified graph of FF according to other reports (Holton and Cornish, 1995; Lillo et al., 2008; Winkel-Shirley, 2002) is as follows (Fig. 1). Flavonoids are derivatives of the products of the shikimate and the acetate-malonate pathways. The central step in FB is the condensation of three molecules of malonyl-CoA with a molecule of $p$-coumaroyl-CoA to yield the $\mathrm{C}_{15}$-chalcone intermediate (naringenin chalcone), a reaction catalyzed by chalcone synthase (CHS). This intermediate is further transformed into various flavonoids. 


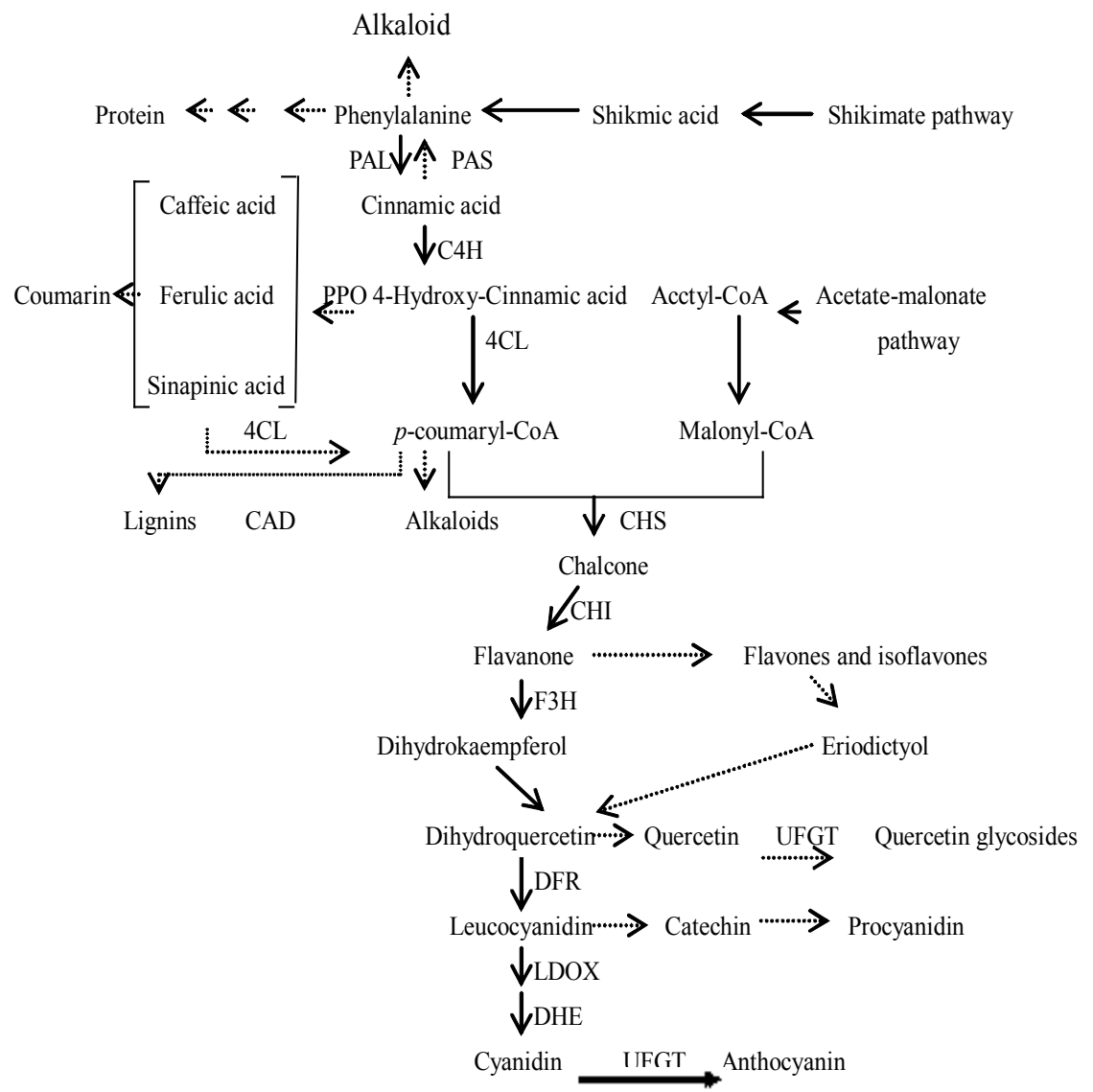

Fig. 1. Putative biosynthetic pathway to anthocyanin and flavonoids in plants

The first sources of flavonoids are the products of photosynthesis. Their precursors are simple phenols. PAL catalyzes the deamination of ${ }_{L}$-phenylalanine to yield transcinnamic acid and ammonia, which is the first committed step in the biosynthesis of the phenylpropanoid skeleton. PAL activity is closely related to the physiological or developmental status of plant. In many cases, the increase in PAL is also accompanied by the appearance of other enzymes associated with the FB pathway (Ferrer et al., 2008; Markus et al., 2008). However, many other products, such as anthocyanin, cutin, and lignin, are biosynthesized during the course of conversion of phenolic compounds into flavonoids. Therefore, FF is sometimes not dependent on PAL activity.

$\mathrm{CHS}$, the first enzyme in the FB pathway, is responsible for the establishment of the $\mathrm{C}_{15}$ skeleton of flavonoid compounds. Its activity is related to light intensity, and it is considered a key enzyme regulating FB (Müller et al., 2010; $\mathrm{Xu}$ et al., 2007; Zifkin et al., 2012). Moreover, 4-coumaroyl-coenzyme A ligase ( $4 \mathrm{CL}$ ), having many isoenzymes, which lies at a branch point from which many different types of products are derived, is also regarded as an important enzyme. In addition, UDP-glucose:flavonoid 3-Oglucosyltransferase (UFGT) catalyzing the formation of stable anthocyanin from unstable anthocyanidin and the formation of quercetin glycosides from quercetin, is closely correlated with the biosynthesis and transformation of flavonoids and anthocyanin (Ju et al., 1995). Isomerization and transformation of flavonoids often occur spontaneously without chalcone isomerase $(\mathrm{CHI})$, but these processes can be accelerated by CHI (Cheng et al., 2010).

Enzymes are key factors involved in FB. In the case of $\mathrm{FB}$ in G. biloba leaf, the two main questions are: What are the limiting enzymes? What relative components are the most important? Based on the above questions, the FC and other related components and enzymes in G. biloba leaf were determined during all the growth stages. By comparative analysis, the key enzymes in FB were proposed, and the components closely related to FB were found, which provided the theoretical foundation for the regulation of FB and enhancement of FC.

\section{Materials and methods}

\section{Plant material and experimental design}

Seven-year-old trees of the G. biloba cultivar 'Jiafoshou', grafted onto a domestic seedling stock of $G$. biloba cultivar 'Dong Ting Huang', were selected for this study. Standard commercial culture methods were used in all the experiments, unless otherwise indicated.

The leaves used for determining the components and the enzyme activities were obtained through random sampling from the phyllome branches of in G. biloba trees in the east, south, west, and north directions. The contents 
100

of lignin, anthocyanin, and simple phenols, FC, and the activities of PAL, CHS, and UFGT in the control were analyzed during the period from May 4 to November 29, 2009. Dry leaf samples were subjected to composition analysis, and fresh leaf samples were used for the assay of enzyme activity or stored at $-20^{\circ} \mathrm{C}$. The samples were selected from random group design with three repetitions. The estimation of internal PAL and CHS activities in the leaves was conducted on a monthly basis from July $18^{\text {th }}$ to November $29^{\text {th }}$.

Flavonoids, anthocyanin, simple phenol, and soluble protein

The analysis of $\mathrm{FC}$ was carried out using the methods described in literature (Xu et al., 2007). Two hundred milligrams of powder of dry leaf were mixed with $10 \mathrm{~mL}$ $70 \%(\mathrm{~V} / \mathrm{V})$ alcohol of the incubated at $37^{\circ} \mathrm{C}$ for $3 \mathrm{~h}$, and the homogenate was centrifuged at $19000 \mathrm{~g}$ for $20 \mathrm{~min}$. Subsequently, and $20 \mu \mathrm{L}$ of the supernate were added to $10 \mu \mathrm{L}$ of a solution of $2 \%$ 4-dimethylaminocinnamaldehyde (DMACA) solution in a mixture of equal volumes of methanol and $\mathrm{HCl} 6 \mathrm{~mol} / \mathrm{L}(\mathrm{V} / \mathrm{V})$ and to $970 \mu \mathrm{L}$ methanol. After $3 \mathrm{~h}$ in darkness, the absorbance at $637 \mathrm{~nm}$ was measured with a recording spectrophotometer (KONTRON UV 941). The final data were analysed statistically (values were each the mean of three to ten replicates) and were expressed as percentage.

Anthocyanin content was determined according to $\mathrm{Xu}$ et al. (2008b) and expressed as $\mathrm{nMol} / \mathrm{cm}^{2}$. Protein concentration was determined using the binding assay of Bradford (1976), with bovine serum albumin as the standard (the same procedure was followed for enzymes).

Simple phenol content was assayed by the methods of Pirie et al. (1976), with some improvement. A small amount $(0.15-0.2 \mathrm{~g})$ of dry powder was mixed with methanol containing $1 \% \mathrm{HCl}$. After grinding and complete mixing, the solution was made up to $25 \mathrm{ml}$ and stored in the refrigerator $\left(0^{\circ} \mathrm{C}\right)$ for 24 hours. After centrifugation, the supernatant fluid was used for estimation of simple phenols. The absorbance of simple phenols was measured at 280,530 , and $600 \mathrm{~nm}$. One unit of simple phenol content $(1 \mathrm{U})$ was defined as a net change of 0.1 in the value of $A_{280}\left(A_{530} A_{600}\right) \times 63 \%$. The result was expressed as $U / g$ dry weight.

Lignin analyses were carried out on dry leaf powder, and ground to pass through a $0.5-\mathrm{mm}$ sieve, before exhaustive solvent extraction $(2: 1[\mathrm{v} / \mathrm{v}]$ toluene: ethanol, ethanol and then water). The lignin content of plant leaves was determined by the Klason lignin method (Dence and Lin, 1992).

\section{Assays of enzyme activities}

Assay of PAL activity was conducted according to the method of Xu et al. (2008a). Fresh leaves, cut into many snippets and weighing approximately 0.2-0.3 g, were mixed with $5 \mathrm{ml}$ of $0.1 \mathrm{~mol} / \mathrm{L}$ boric acid buffer (with $1 \%$ polyvinylpyrrolidone (PVP), $\mathrm{pH} 8.8$ ); ground, extracted, and filtered; and centrifuged at $13,000 \times g$ for 10 minutes. The supernatant fluid was used for the assay of PAL activity. All operations were carried out under low temperature, and the operation conditions for other enzymes were similar to those of PAL. One unit of enzyme activity $(1 \mathrm{U})$ was defined as the amount of enzyme required for a change of 0.01 units in the absorbance at $295 \mathrm{~nm}$ per hour under the assay conditions. PAL activity was expressed as $\mathrm{U} \cdot \mathrm{mg}^{-1}$ protein.h ${ }^{-1}$.

Assay of polyphenol oxidase (PPO) activity was carried out according to the method of Yang et al. (1997), with some modifications. Fresh leaves, which were cut into pieces and weighed $0.4-0.5 \mathrm{~g}$, were mixed with $5 \mathrm{ml}$ of 0.05 $\mathrm{mol} / \mathrm{L}$ citrate-phosphate buffer $(\mathrm{pH}$ 6.8) and $0.1 \mathrm{~g}$ PVP. After grinding, extraction, and filtration, the mixture was centrifuged $(10$ minutes at $13,000 \times g)$, and the resulting supernatant was used for the assay of PPO activity. One unit of enzyme activity ( $1 \mathrm{U}$ ) was defined as the amount of enzyme required for a change of 0.0001 units in the absorbance at $525 \mathrm{~nm}$ per minute under the assay conditions. PPO activity was expressed as $\mathrm{U} \cdot \mathrm{mg}^{-1}$ protein $\cdot \mathrm{min}^{-1}$.

Peroxidase (POD) activity was assayed according to the method described by Park (2006), with some modifications. Many steps in the determination of POD activity were similar to the assay for PPO, except that 0.1 $\mathrm{mol} / \mathrm{L}$ Tris- $\mathrm{HCl}$ buffer $(\mathrm{pH} 8.5)$ was used and PVP was not used. One unit of enzyme activity $(1 \mathrm{U})$ was defined as the amount of enzyme required for a change of 0.01 units in the absorbance at $470 \mathrm{~nm}$ per minute under the assay conditions. POD activity was expressed as U.mg ${ }^{-1}$ protein. $\mathrm{min}^{-1}$.

Extraction and assay of the activities of UFGT and CHS were according to the report by Ju et al. (1995). Fresh fragmented G. biloba leaves, weighing approximately 5-15 g, were selected at different growth and developmental stages. One unit (1 U) of UFGT activity was defined as the amount of enzyme required for the formation of $1 \mathrm{~mol}$ of quercetin-3-galactoside per second under the assay conditions. The activities of UFGT and CHS were expressed as $\mathrm{U} \cdot \mathrm{mg}^{-1}$ protein $\cdot \mathrm{s}^{-1}$ and $\mathrm{U} \cdot \mathrm{mg}^{-1}$ protein $\cdot \mathrm{min}^{-1}$, respectively.

Every result was obtained as the average of three tests. Statistical analysis of data and table design used the DPS and Microsoft Excel softwares.

\section{Results}

FC and contents of anthocyanin and simplephenols in $G$. biloba leaves and their relationships

FC showed two obvious peaks of in G. biloba leaves (Tab. 1). The dates of the peak and the peak values were Jul $4^{\text {th }}(0.8082 \%)$ and Nov. $1^{\text {st }}(1.0625 \%)$, respectively. The latter peak value was higher than the former one by $19.09 \%$ and remained high for a longer duration. In other words, FC remained at a high level from September 29. When the 
highest peak appeared during Nov., the net change of FC was less than $1.5 \%$ in the first fifteen days. Furthermore, the FC decreased by only approximately 3\% in Nov. All the above observations showed that FC remained stable during the second synthesis peak in Nov, which is hence an optimal period to collect $G$. biloba leaves.

The change in anthocyanin content was larger and with greater fluctuation, which was similar to that of FC from the viewpoint of the total direction of change. In terms of relative percent increase in the content, it was found that anthocyanin content was lower than FC during the early and late stages and was higher than FC during the middle stage, respectively. During the course of conversion of simple phenols into flavonoids, including anthocyanin, FB predominantly synthesizes anthocyanin during the early and late stages. If the intensity of synthesis is regarded as a comparative index, and the other compounds are synthesized during the middle stage. The results also indicated that there was a rhythm, a time-distribution character, to the synthesis and metabolism of flavonoids and anthocyanin in plants.

During the early stages, the content of simple phenols and FC increased concurrently and both reached their first peaks on Jul. $4^{\text {th }}$. After content of simple phenols decreased to the lowest value on Aug. $1^{\text {st }}$, it again increased rapidly. The peak period of simple phenol synthesis was from midAug. to late Sep., and the simple phenol content remained at a high and stable level during this period. During early Aug., the decrease in simple phenol content was perhaps related to lignin synthesis. However, after late September, the steep decrease in simple phenols resulted from an increased synthesis of flavonoids and lignin (Tab. 1).

During the early and late stages, soluble protein content was at a higher level when FC was at a lower level. However, there was a positive and significant correlation between these two from Jul. $18^{\text {th }}$ to Sept. $29^{\text {th }}$, with $r=$ 0.9354, and the regression equation was as following: $Y$ (soluble protein $)=47.0635 X$ (flavonoids) -16.5742 ; adjusted $\mathrm{R}^{2}=0.8437$. This indicated that the relationship between soluble proteins and flavonoids varied with time.

\section{Comparison of PPO, POD, and PAL activities}

Three obvious peaks of PPO activity were found in $G$. biloba leaves (Fig. 2). The dates of the peak and the peak values were Jul. $4^{\text {th }},(3.74 \mathrm{U})$, Aug. $30^{\text {th }}(3.06 \mathrm{U})$, and Oct. $17^{\text {th }}(7.93 \mathrm{U})$, respectively. Of all the peaks, the value of the third peak was the highest and its gradient was the steepest. The PPO activity was the lowest on July $27^{\text {th }}$, with the value being $0.69 \mathrm{U}$.

POD activity always fluctuated based on its total change trend. Furthermore, PPO activity remained at a lower level before Sep. $11^{\text {th }}$, and its lowest value $(0.20 \mathrm{U})$ appeared on Jun. $13^{\text {th }}$. From Sep. $11^{\text {th }}$ onward, POD activity increased rapidly and reached its highest peak on Oct. $17^{\text {th }}(12.16 \mathrm{U})$. There was 61 -fold difference, between the highest and the lowest values, which probably was related to the POD isoenzymes and their total activity assayed in this study. This result also indicated that POD activity had little effect on phenol content or that the lower POD activity met the normal demands of metabolism during the early and middle stages.

Three peaks of PAL activity were found during the course of a year, and the dates of the peaks and their values were Jul. $18^{\text {th }}(4.99 \mathrm{U})$, Aug. $30^{\text {th }}(2.61 \mathrm{U})$, and Nov. $14^{\text {th }}(3.54 \mathrm{U})$, respectively. PAL is the first enzyme in the biosynthesis of a wide range of phenylpropanoid compounds, such as simple phenols, anthocyanin, flavonoids, and lignin. Similarly, PPO and POD are closely correlated with the process of lignin synthesis (Uzal et al., 2009). In other words, PPO, POD, and PAL, directly or indirectly,

Tab. 1. The seasonal patterns of the contents of flavonoids, anthocyanin, simple phenols, lignin and soluble protein in Ginkgo biloba leaves

\begin{tabular}{cccccc}
\hline Date & $\begin{array}{c}\text { Flavonoids } \\
\text { \% DW }\end{array}$ & $\begin{array}{c}\text { Anthocyanin } \\
\mathrm{nMol} \cdot \mathrm{cm}^{-2}\end{array}$ & $\begin{array}{c}\text { Simple phenols } \\
\mathrm{U}(\mathrm{g} \mathrm{DW})^{-1}\end{array}$ & $\begin{array}{c}\text { Lignin } \\
\% \mathrm{DW}\end{array}$ & $\begin{array}{c}\text { Soluble protein } \\
\mathrm{mg} \cdot \mathrm{g}^{-1}\end{array}$ \\
\hline 4-May & $0.3125 \pm 0.0104$ & $0.2244 \pm 0.0085$ & $111.43 \pm 7.58$ & $30.24 \pm 1.96$ & $19.11 \pm 0.57$ \\
18-May & $0.5113 \pm 0.0097$ & $0.3451 \pm 0.0102$ & $176.03 \pm 11.87$ & $32.25 \pm 1.06$ & $9.03 \pm 0.46$ \\
13-Jun & $0.6410 \pm 0.0215$ & $0.3537 \pm 0.0105$ & $218.56 \pm 12.38$ & $31.42 \pm 2.10$ & $12.18 \pm 0.84$ \\
4-Jul & $0.8082 \pm 0.0173$ & $0.4256 \pm 0.0121$ & $369.47 \pm 14.62$ & $32.24 \pm 1.53$ & $10.68 \pm 0.78$ \\
18-Jul & $0.7124 \pm 0.0260$ & $0.4817 \pm 0.0092$ & $352.10 \pm 11.25$ & $32.50 \pm 2.88$ & $7.89 \pm 0.25$ \\
\hline 27-Jul & $0.6369 \pm 0.0155$ & $0.3188 \pm 0.0137$ & $329.77 \pm 19.24$ & $35.07 \pm 2.64$ & $14.53 \pm 0.50$ \\
1-Aug & $0.6767 \pm 0.0169$ & $0.6654 \pm 0.0295$ & $168.55 \pm 10.80$ & $32.61 \pm 2.42$ & $13.57 \pm 0.62$ \\
13-Aug & $0.7784 \pm 0.0483$ & $0.6024 \pm 0.0220$ & $453.54 \pm 33.61$ & $33.45 \pm 1.58$ & $18.79 \pm 1.02$ \\
30-Aug & $0.7271 \pm 0.0402$ & $0.7106 \pm 0.0434$ & $410.07 \pm 28.76$ & $33.30 \pm 2.05$ & $16.40 \pm 0.96$ \\
\hline 11-Sep & $0.7271 \pm 0.0220$ & $0.5771 \pm 0.0128$ & $433.25 \pm 18.39$ & $34.69 \pm 1.32$ & $20.30 \pm 0.88$ \\
29-Sep & $0.9125 \pm 0.0177$ & $0.6989 \pm 0.0216$ & $424.30 \pm 12.65$ & $37.54 \pm 1.19$ & $26.81 \pm 0.54$ \\
17-Oct & $0.9057 \pm 0.0204$ & $0.6239 \pm 0.0325$ & $201.89 \pm 9.78$ & $39.55 \pm 1.26$ & $18.44 \pm 0.61$ \\
01-Nov & $1.0625 \pm 0.0158$ & $0.5489 \pm 0.0274$ & $362.23 \pm 24.84$ & $34.67 \pm 1.13$ & $15.91 \pm 0.92$ \\
14-Nov & $1.0465 \pm 0.0181$ & $0.6868 \pm 0.0299$ & $427.38 \pm 15.33$ & $35.99 \pm 2.20$ & $23.47 \pm 0.86$ \\
29-Nov & $1.0306 \pm 0.0396$ & $0.4664 \pm 0.0124$ & $439.74 \pm 23.69$ & $36.88 \pm 1.41$ & $15.45 \pm 0.72$ \\
\hline
\end{tabular}




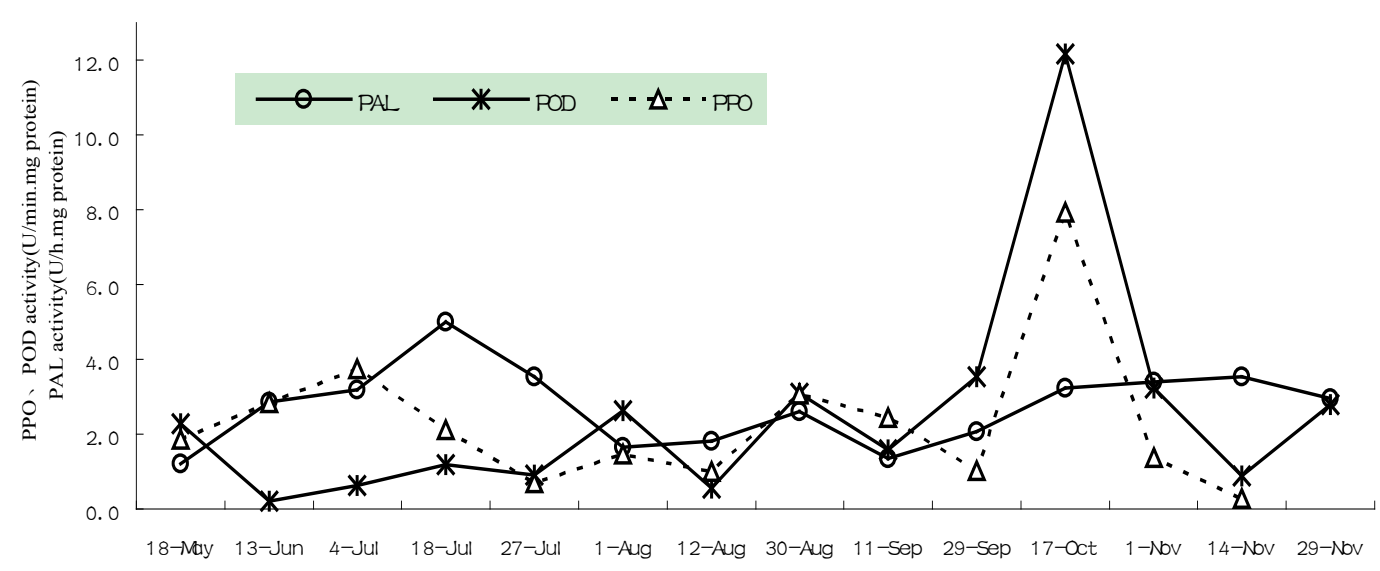

Fig. 2. The seasonal patterns of POD, PPO, PAL activities of Ginkgo biloba leaves

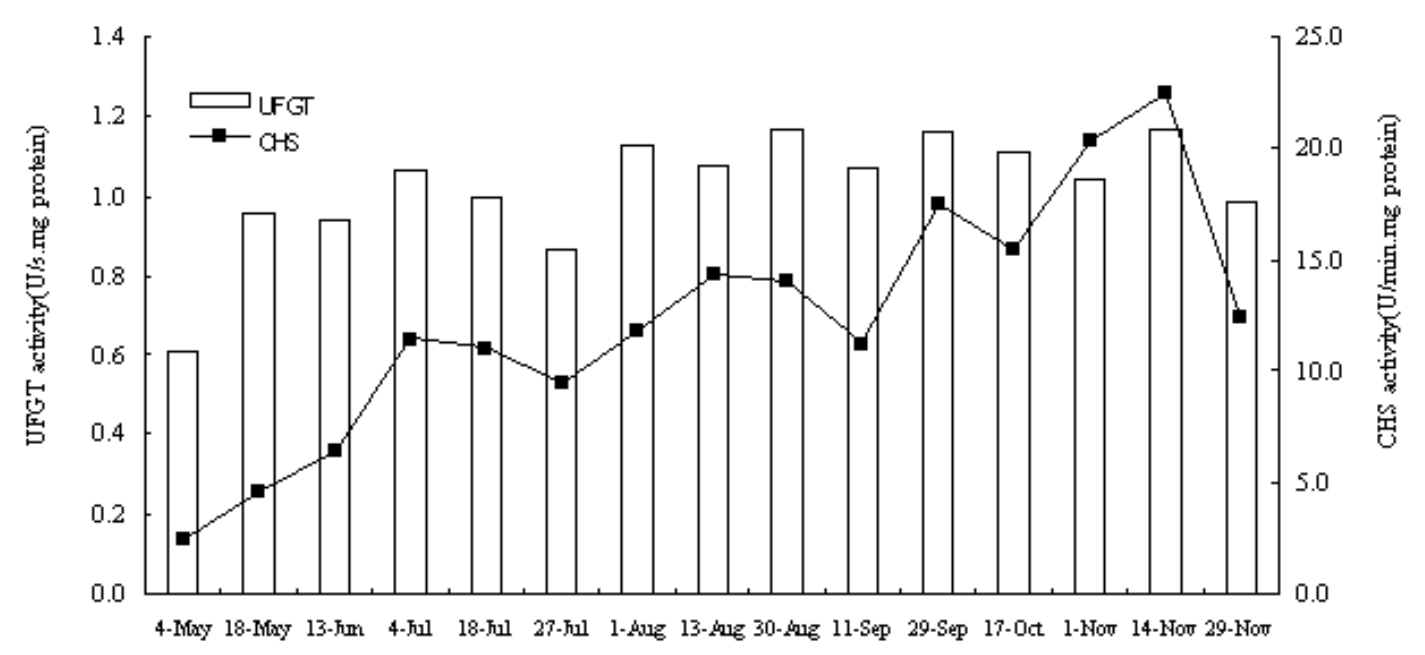

Fig. 3. The seasonal patterns of UFGT and CHS activities in Ginkgo biloba leaves

affect FC by influencing the synthesis of other secondary metabolites and thus regulate $\mathrm{FB}$ during its initial course. Comparing the changes in the activities of PPO, POD, and PAL, PPO and PAL activities increased simultaneously during the early stages, and the first activity peak of PPO showed up before that of PAL. However, PAL activity proceeded to a higher level when compared with PPO activity. POD activity remained at a low level, with much fluctuation. Their activities reached a lower peak on Aug. $8^{\text {th }}$. After Sep. $11^{\text {th }}$, while PAL activity increased steadily and remained at a high level for a long duration, PPO and POD activities fluctuated rapidly with large increases and decreases. The final peak activities of PPO and POD were reached on Oct. $17^{\text {th }}$, whereas that of PAL was on Nov. $14^{\text {th }}$.

\section{Changes of UFGT and CHS activities}

UFGT activity increased rapidly, reached a higher level on May $18^{\text {th }}$, and then remained at this high level, in spite of the fluctuation in its activity and one valley of lesser activity on Jul. $27^{\text {th }}$. CHS activity increased gradually during the early stage and reached the first peak on July 4. It showed an increasing trend, with a small slope during the middle stage. After Sept. $11^{\text {th }}$, the CHS activity increased again with a large slope, reached its highest value on Nov. $14^{\text {th }}$, and thereafter decreased steeply (Fig. 3).

The results are summarized as follows by comparing the changes in the activities of the five enzymes. POD activity is maintained at a low level during the early and middle stages. However, its activity fluctuates rapidly and considerably. UFGT activity increases sharply during the early stage and then remains relatively constant at a high level. In general, CHS activity increases constantly before Nov. $14^{\text {th }}$ and falls sharply subsequently. PAL activity is higher in the early and late stages than in the middle stage, with the highest activity level occurring during the early stage. However, its activity remains high throughout the late stage. The change in PPO activity can be represented by a three-peak curve, with an activity peak during the early, middle, and late stages.

\section{Relationship between enzyme activities and related components}

Comparing the changes in the PAL activity and FC (Tab. 1 and Fig. 2), a similar trend could be found, especially in the two FC peaks during the early and last stages. Nevertheless, linear regression analysis indicated that there was no significance correction between PAL and FC. The 


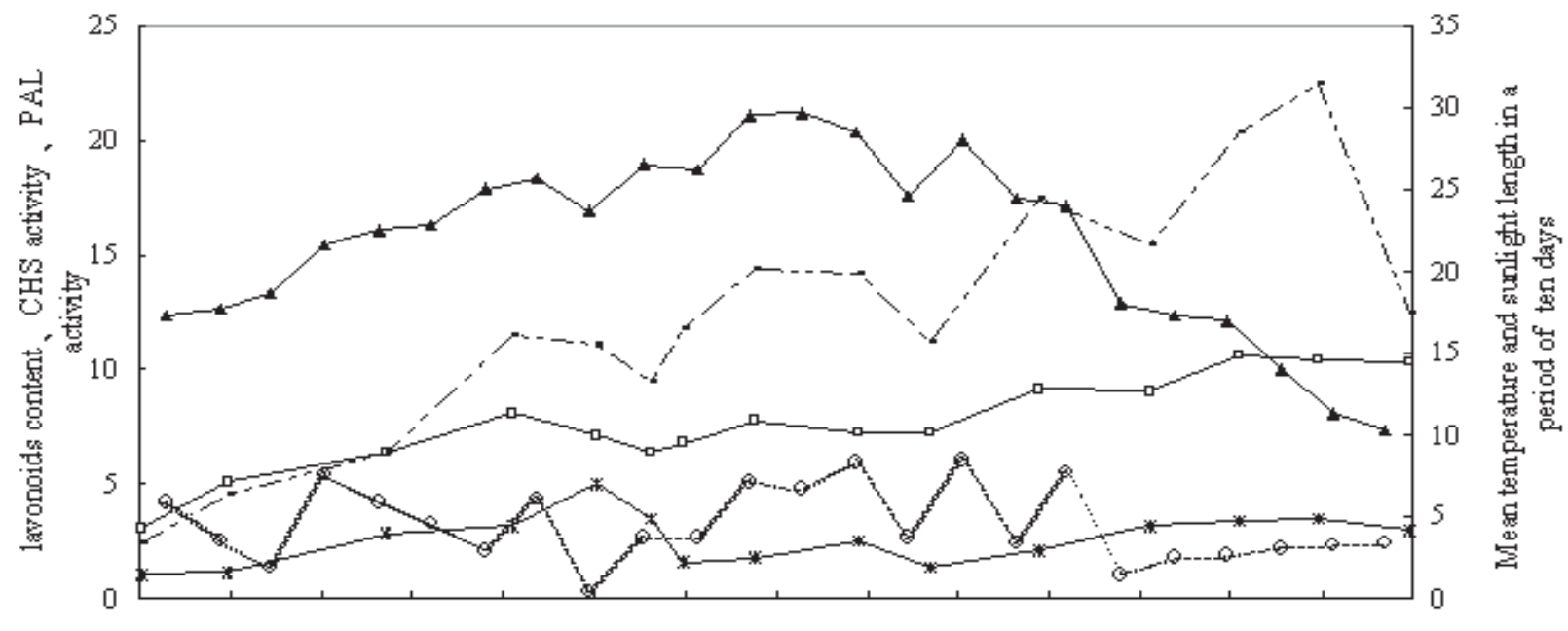

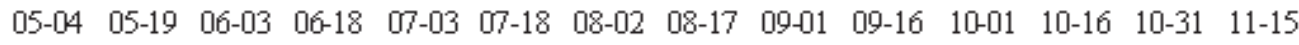

Date (rmm-dd)
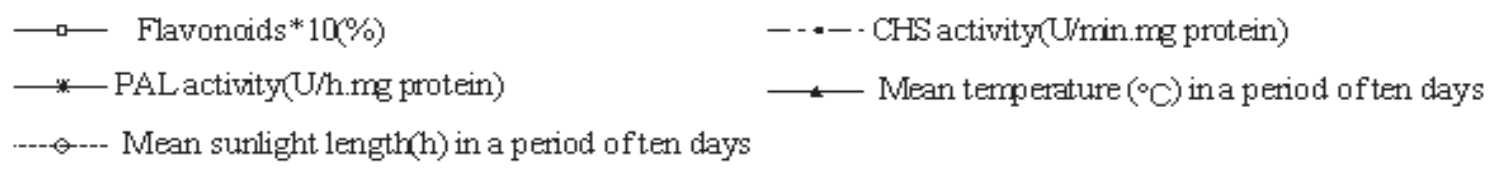

Fig. 4. The seasonal patterns of the mean temperature and sunlight length in a period of ten days, flavonoids content, CHS and PAL activity of Ginkgo biloba leaves

reasons are as follows. First, although their activity or content increase almost simultaneously, several peaks appear on different dates. The peaks corresponding to PAL activity always lagged behind the FC peaks, which probably is related to the synthesis of other secondary metabolites (Chenget al., 2009). Second, there was no similarity in the trend of change between PAL activity and FC during the two periods of the middle stage. Contrarily, FC increased to some extent, whereas PAL activity decreased. Based on the analysis of data (May $4^{\text {th }}-$ Jul. $4^{\text {th }}$ and Sept. $11^{\text {th }}-$ Nov. $\left.29^{\text {th }}\right)$, the correlation coefficient $(0.7964)$, the adjusted $\mathrm{R}^{2}$ (0.5886), and the regression equation were obtained, that is $Y$ (flavonoids) $=0.2876+0.2044 X(\mathrm{PAL})$, which indicated a significant correction between FC and PAL activity. Altogether, PAL activity played an important role in the FB peak observed during the early and final stages and the stages when regulation of PAL activity is useful to increase the FC.

CHS, having a close relationship with flavonoids including anthocyanin, is the first enzyme that catalyzes FB. The relationships of CHS with the other components are as follows: (1) CHS and flavonoids: correlation coefficient $(r)=0.8952$, adjusted $\mathrm{R}^{2}=0.7861$, regression equation: $Y$ (flavonoids) $=0.3478+0.0339 X(\mathrm{CHS})$, and F-test is statistically significant at the $1 \%$ level. On further analysis, almost the same change trend between CHS and flavonoids is observed before mid-Aug., and the correlation coefficient $(r)=0.9051$, regression equation: $Y$ (flavonoids) $=$ $0.0352 X(\mathrm{CHS})+0.3194$. They both reached significance at the $1 \%$ level when analyzed by the F-test. However, there was no significant relationship between them after mid-Aug $\left(r=0.6600, r_{0.05}=0.7540\right)$. The relationship between FC and CHS activity changed at different times of the year. In other words, the effect of CHS on FC before mid-Aug was greater than that after mid-Aug. (2) CHS and anthocyanin: The relationship between CHS and anthocyanin is similar to the relationship between CHS and flavonoids, and the difference is only that the correlation coefficient and the adjusted $\mathrm{R}^{2}$ between CHS and anthocyanin are relatively lower than those between CHS and flavonoids $\left(r=0.8031, \mathrm{R}^{2}=0.6167\right)$. Regression equation, $Y($ anthocyanin $)=0.2329+0.0229 X(\mathrm{CHS})$ reached statistical significance at the $1 \%$ significance level. (3) CHS and flavonoids + anthocyanin: CHS catalyzes the conversion of simple phenols to flavonoids and then into anthocyanin in many steps. Thus, a close relationship exists among these components. Based on the analysis of correlation and regression, the correlation coefficient (0.9451), the adjusted $\mathrm{R}^{2}(0.88490)$, and the regression equation, $Y$ (flavonoids + anthocyanin $)=0.5807+0.0568 X(\mathrm{CHS})$, which reached the $1 \%$ significant level by the F-test. Of all the indexes, the values of the correlation coefficient and the adjusted $\mathrm{R}^{2}$ were greater than the above corresponding indexes, which further proved that there was a virtually homologous relationship between flavonoids and anthocyanin (Makoia et al., 2010). Meanwhile, the results indicated that using CHS to forecast the total FC and anthocyanin content is more desirable than to forecast the FC and the anthocyanin content separately. During the near-defoliation stage, when FC decreased, CHS activ- 
104

ity increased, and anthocyanin content also changed from a lower to a higher level. Thus, the relationship between CHS, flavonoids, and anthocyanin is explained with the total content of anthocyanin and FC, which disproves the result of the above regression analysis.

\section{Discussion}

Phenylpropane metabolism is an important path that yields all types of secondary substances in plants. Phenol synthesis originates from phenylalanine and is catalyzed by PAL. More than ten types of enzymes take part in the synthesis of flavonoids, including anthocyanin, from phenylalanine. Furthermore, PAL, 4CL, cinnamic acid-4hydroxylase $(\mathrm{CA} 4 \mathrm{H}), \mathrm{CHS}$, and UFGT are key enzymes in FB. Thus, PAL was selected as the representative for PAL, 4CL, and CH4A due to the following reasons. (1) There was a concomitant relationship between PAL activity and the activities of $4 \mathrm{CL}$ and $\mathrm{CA} 4 \mathrm{H}$. (2) The factors that inhibit PAL activity can also limit 4CL and CA4H activities, and vice versa. For example, ethylene can promote PAL activity, in addition to enhancing CA4H and 4CL activities (Ju et al., 1995; Roura et al., 2008). In addition, PPO and POD were also studied based on their special effects on phenols. Therefore, in total, PAL, CHS, UFGT, POD, and PPO were investigated in this study. PAL is the first enzyme in phenol synthesis, and its activity was almost parallel to the total content of simple phenols in the early stage. The FC, with simple phenols as precursors, increased simultaneously. However, this results was related with the lower POD activity (Forkmann, 1991). The content of simple phenols and FC have been previously reported to be decreased, in addition to anthocyanin being decomposed, when POD activity increases (Du et al., 1997). In other words, the lower POD activity causes simple phenols to be transformed into flavonoids, including anthocyanin. Although PPO had some activity and lignin was synthesized to some extent, they were found at lower levels, which was also a precondition for the gradual increase of flavonoid levels derived from simple phenols. FB reached its first synthesis peak in mid-July when CHS activity increased. After mid-July, PAL activity decreased sharply and remained at a lower level, while the simple phenol content increased. The accumulation of simple phenols resulted from the low activities of CHS, UFGT, PPO, and POD, in addition to the metabolism of inactive secondary substances. However, PAL first entered the active period from the beginning of Sep., then CHS became active, and finally, POD and PPO increased and decreased, respectively, with great intensity. These results can be explained by considering the contents of the products. Because other secondary substances are synthesized in large amounts, the simple phenol contents decreased sharply in spite of the increase of PAL activity. The change in lignin content was parallel to that of the POD and PPO activities, which was consistent with the results of previous re- ports ( $\mathrm{Du}$ et al., 1997). The increase in the interference by PPO and POD resulted in the FC, including anthocyanin content, increasing insignificantly or even slowly, although their syntheses were accelerated during this period of active FB. However, after the PPO and POD activities decreased sharply, the FC increased rapidly and reached the highest peak value if the PAL activity remained at a higher level. The FC, as one of the products, declined finally when the activities of all the above enzymes decreased. PPO has been reported to promote FB (Cheng et al., 2009), which is not in accordance with the results of this study. Further research will be necessary to determine whether different materials resulted in these differences.

The activity of UFGT increased sharply and reached a higher level during the early stages and then remained stable, which probably indicated that UFGT had no significant effect on FB during the middle and late stages of leaf development. Simple phenols could normally change into flavonoids and anthocyanin. This phenomenon is especially conspicuous in the case of anthocyanin. The trend of change in anthocyanin content, with little fluctuation, was similar to that of UFGT activity during the middle and late stages. These results clarify that although UFGT was virtually related to anthocyanin synthesis, the relationship between UFGT and anthocyanin synthesis in different organs was different, such as leaf and fruit. Fruit peels were selected as test materials in former studies. The peels rapidly became reddish in color because the chlorophylls and carotenoids decomposed, in addition to anthocyanin being synthesized rapidly, during the maturing of fruits, which resulted from the large increase of UFGT activity and the resulting decrease in FC ( $\mathrm{Hu}$ et al., 2011). Compared with fruit peels, the leaves became light yellow or yellow gradually because of the decomposition of chlorophylls and the process of $\mathrm{FB}$, while anthocyanin content remained at the former level or was synthesized in very little amounts. Therefore, anthocyanin content can be regarded as an external indicator due to the difference in the color-change pattern between the peel and the leaf during the late stage. Of course, further work needs to be carried out to determine whether UFGT directly takes part in the conversion of flavonoids.

Compared with the other enzymes, CHS plays a key role in FB. The curves representing the changes in CHS activity and FC were almost synchronous. However, the slight differences observed maybe due to conversion of flavonoids into anthocyanin by the action of UFGT (Zhang et al., 1995). Based on all the above results, the conclusions were that CHS is the first key enzyme in FB and that higher PAL activity was the precondition for the large-scale synthesis of flavonoids. In addition, PPO and POD had some influences on FF, whereas UFGT seems to have no effect on FB.

To elucidate the relationships between the enzymes, flavonoids, and weather, all the relevant data are shown in Fig. 4. When the mean temperature increased over 
a period of ten days during the early stage, PAL activity increased, as well as the FC. The optimal temperature of PAL activity and $\mathrm{FB}$, including anthocyanin synthesis, was previously shown to be $16-25^{\circ} \mathrm{C}$ ( Ju et al., 1992; Xu et al., 2008a), which agreed with the results of this study in $G$. biloba leaf. However, when the mean temperature exceeded $25^{\circ} \mathrm{C}$ during Jul. and Aug., FC remained at a low and stable level. Because of the sharp decrease in temperature, by approximately $10^{\circ} \mathrm{C}$ in Oct., compared with that in Sep., as a protective reaction of G. biloba leaf to temperature change, the PAL activity rapidly increased, with a concomitant increase in FC, which was also consistent with our previously work (Wang et al., 2007). The relationship between $\mathrm{CHS}$ and temperature was similar to that between PAL and temperature, the only difference being that CHS seemed to be more tolerant to high temperature and more sensitive to low temperature, which may result from the characteristics of the $\mathrm{CHS}$ and PAL enzymes themselves. This observation indicated that the effect of temperature on enzyme activity resulted in an effect on FB. To regulate FB through enzyme activity is thus a good idea to practice.

\section{Conclusions}

PPO and PAL both had three activity peaks, with the largest value in the third peak. The changes in PAL activities accompanied the changes in FC during the early and later stages. POD activity was at a low level during the early stage, increased rapidly after mid-September, and reached the highest value in mid-October. This trend was almost parallel to the trend of change in lignin content. Especially during the later stage, there was a significant correlation between POD activity and lignin content. Simple phenol content reached the highest level when PAL, PPO, and $P O D$ retained their higher activities during the end of August. UFGT activity increased sharply and reached a higher level during the early stage and then remained stable. As a whole, CHS activity increased at all times of the year, with the correlation between CHS and FC reaching a $1 \%$ significant level. In conclusion, $\mathrm{CHS}$ is the first key enzyme in FB, and PAL plays an important role during the early and later stages of G. biloba leaf growth and development. PPO and POD both have some effect on FB through their regulation of levels of simple phenols, and UFGT seems to have no effect on FB. The influence of temperature on $\mathrm{FB}$ is conspicuous through its regulation of the related enzymes, such as CHS and PAL. The overall aim of present work is to provide theoretical and practical bases for increasing the flavonoid content of G. biloba leaves for commercial production.

\section{Acknowledgements}

This work was supported by the National Natural Science Foundation of China (309719743, 31000904), the Key Project of Chinese Ministry of Education (No.
212112), the Open Fund of Hubei Key Laboratory of Economic Forest Germplasm Improvement and Resources Comprehensive Utilization (20011BLKF240), Natural Science Foundation of Hubei Province (2009CDB232), and University-Industry Cooperation Fund of Hubei Educational Office (CXY2009B009).

\section{References}

Bradford MM (1976). A rapid and sensitive method for the quantitation of microgram quantities of protein utilizing the principle of protein-dye binding. Anal Biochem 72:248254.

Cheng H, L Li, Cheng S, Cao F, Wang Y and Yuan H (2011). Molecular cloning and function assay of a chalcone isomearse gene (GbCHI) from Ginkgo biloba. Plant Cell Rep 30:4962.

Cheng SY, Xu F, Wang Y (2009). Advances in the study of flavonoids in Ginkgo biloba leaves. J Med Plants Res 3:12481252.

Dence CW, Lin SY (1992). The determination of lignin, 3361 p. In: Lin SY, Dence CW (Eds.). Methods in lignin chemistry. Springer-Verlag, Berlin.

Du JH, Zhang KL, Guo Y (1997). Anthocyanin production by plant cell culture technique. J Shangdong Agri Univ 28:511517.

Ferrer JL, Austin MB, Stewart C, Noel JP (2008). Structure and function of enzymes involved in the biosynthesis of phenylpropanoids. Plant Physiol Biochem 46:356-370.

Forkmann G (1991). Flavonoids as flower pigments: the formation of the natural spectrum and its extension by genetic engineering. Plant Breed 106:1-26.

Müller GL, Drincovich MF, Andreo CS, Lara MV(2010). Role of photosynthesis and analysis of key enzymes involved in primary metabolism throughout the lifespan of the tobacco flower. J Exp Bot 61:3675-3688.

Hao G, Du X, Zhao F, Ji H (2010). Fungal endophytes-induced abscisic acid is required forflavonoid accumulation in suspension cells of Ginkgo biloba. Biotechnol Lett 32:305314.

Holton TA, Cornish EC (1995). Genetic and biochemistry of anthocyanin biosynthesis. Plant Cell 7:1071-1083

Hu CY, Gong YF, Jin S, Zhu Q (2011). Molecular analysis of a UDP-glucose: flavonoid 3-O-glucosyltransferase (UFGT) gene from purple potato (Solanum tuberosum). Mol Biol Rep 38:561-567.

Ju ZG, Liu CL, Yuan YB (1993). Regulation of phenolics synthesis and its effects on fruit quality of Laiyang Chili (pear). Sci Agri Sin 26:44-48.

Ju ZG, Liu CL, Yuan YB (1995). Activities of chalcone synthase and UDPGal: flavonoid-3-0- glycosyltransferase in relation to anthocyanin synthesis in apple. Sci Hortic 63:175-185.

Lillo C, Lea U, RuoffP (2008). Nutrient depletion as a key factor for manipulating gene expression and product formation 
106

in different branches of the flavonoid pathway. Plant Cell Environ 31:587-601.

Makoia JH, Belane AK, Chimphango SBM, Dakora FD (2010). Seed flavonoids and anthocyanins as markers of enhanced plant defence in nodulated cowpea (Vigna unguiculata L. Walp.). Field Crops Res 118:21-27.

Markus G, Thomas H, Mari LB, Carlo R, Barbara F, Robert K, Asaph A, Juan MB, Wilfried S (2008). Redirection of flavonoid biosynthesis through the down-regulation of an anthocyanidin glucosyltransferase in ripening strawberry fruit. Plant Physiol 146:1528-1539.

Park S (2006). Study of an enzyme activity in extracts of Ginkgo biloba leaves. Bull Korean Chem Soc 27:1885-1887.

Pirie A, Mullins MG (1976). Changes in anthocyanin and phenolics content of grapevine leaf and fruit tissues treated with sucrose, nitrate, and abscisic acid. Plant Physiol 58:468472.

Rice-Evans AC, Miller NJ, Paganga G (1997). Antioxidant properties of phenolic compounds. Trends Plant Sci 2:152159.

Roura SI, Pereyraa L, Vallea CE (2008). Phenylalanine ammonia lyase activity in fresh cut lettuce subjected to the combined action of heat mild shocks and chemical additives. LWTFood Sci Technol 41:919-924.

Smith JV, Luo Y (2004). Studies on molecular mechanisms of Ginkgo biloba extract. Appl Microbiol Biotechnol 64:465472.

Uzal EN, Gómez Ros LV, Pomar F, Bernal MA, Paradela A, Albar JP, Ros Barceló A (2009). The presence of sinapyl lignin in Ginkgo biloba cell cultures changes our views of the evolution of lignin biosynthesis. Physiol Plant 135:196213.

Van Beek T, Montoro P (2009). Chemical analysis and quality control of Ginkgo biloba leaves extracts, and phytopharmaceuticals. J Chromatogr A 1216(2):20022032.
Wang Y, Li L, Xu F, Liu W, Cheng S (2007). Effects of some metal ions on phenylalanine ammonia-lyase activities and flavonoids content of Ginkgo biloba leaves in the potted orchard. J Nanjing Forest Univ 31:68-72.

Winkel-Shirley B (2002). Biosynthesis of flavonoids and effects of stress. Curr Opin Plant Biol 5:218-233.

Xu F, Cheng SY, Cheng SH, Wang Y, Du HW (2007). Time course of expression of chalcone synthase gene in Ginkgo biloba. J Plant Physiol Mol Biol 33:309-317.

Xu F, Cai R, Du H, Wang Y, Cheng S (2008a). Molecular cloning, characterization and expression of phenylalanine ammonia-lyase gene from Ginkgo biloba. Afr J Biotechnol 7:721-729.

Xu F, Cheng H, Cai R, Li LL, Chang J, Zhu J, Zhang FX, Chen LJ, Wang Y, Cheng SH, Cheng SY (2008b). Molecular cloning and function analysis of an anthocyanidin synthase gene from Ginkgo biloba, and its expression in abiotic stress responses. Mol Cells 26:536-547.

Yang L, Yang SX, Gu ZC(1997). Change pattern of flavones content in Ginkgo biloba growing. J Hubei Forest Sci Technol 3:5-7.

Zhang XQ, Pu FL, Pan GZ (1995). The determination of the total flavonoids content of leaves of Ginkgo biloba in different seasons. J Trad Chinese Med 20:723-724.

Zifkin M, Jin A, Ozga JA, Zaharia LI, Schernthaner JP, Gesell A, Abrams SR, Kennedy JA, Constabel CP (2012). Gene expression and metabolite profiling of developing highbush blueberry fruit (Vaccinium corymbosum L.) fruit indicates transcriptional regulation of flavonoid metabolism and activation of abscisic acid metabolism. Plant Physiol 158: 200-224. 